drive engineered to express an antibody to block an infectious agent would protect people from animal-transmitted disease and maintain native species that are essential ${ }^{9,10}$ to the ecosystem.

Another possible application of mammalian gene drives is to speed the generation of animal models of disease, because it can be challenging to breed a mouse that has specific combinations of mutations in several genes.

Because gene drives have the potential to alter an entire species, appropriate regulation of this technology is a major concern. Only the most intractable and major health challenges should be considered for possible interventions using gene drives. Any proposed genetic change should be tested to minimize the chances of unintended consequences to the species or the ecosystem. This challenge is particularly daunting for highly mobile species such as the mosquito, which can fly long distances and across national boundaries. Certainly, the use of a gene drive for mosquito-borne diseases such as malaria warrants international efforts that proceed using careful planning and monitoring, and with the engagement of local communities. Nevertheless, it should be remembered that even the best-planned efforts can have unexpected outcomes. A mammalian gene drive might offer a more attractive test case than an insect one for pest eradication or infectiousdisease control, because wild mammalian populations can be more easily restricted to a geographic region than can insect populations.

More than 150 years after Mendel's work illuminated one way in which genetic inheritance can be governed, a powerful tool has emerged to manipulate inheritance in mammals. It seems certain that the promise of continual improvements in gene drives will be matched with even more discussion of how to move forward. The development of this technique to generate a mammalian gene drive is another milestone in this exciting area of research..

Bruce R. Conklin is at the Gladstone Institutes, University of California, San Francisco, and at the Innovative Genomics Institute, San Francisco, California 94158, USA. e-mail:bconklin@gladstone.ucsf.edu

1. Mendel, G. J. Verh. Naturforsch. Ver. Brünn 4, 3-47 (1866).

2. McClintock, B. Proc. Natl Acad. Sci. USA 36 344-355 (1950).

3. Gantz, V. M. \& Bier, E. Science 348, 442-444 (2015),

4. Kyrou, K. et al. Nature Biotechnol. 36, 1062-1066 (2018).

5. Grunwald, H. A. et al. Nature 566, 105-109 (2019).

6. Bier, E., Harrison, M. M., O'Connor-Giles, K. M. \& Wildonger, J. Genetics 208, 1-18 (2018).

7. Gantz, V. M. et al. Proc. Natl Acad. Sci. USA 112, E6736-6743 (2015).

8. Jones, H. P. et al. Proc. Natl Acad. Sci. USA 113, 4033-4038 (2016)

9. Bruskotter, J. T., Enzler, S. A. \& Treves, A. Science 333, 1828-1829 (2011).

10.Martinez-Estevez, L., Balvanera, P., Pacheco, J. \& Ceballos, G. PLoS ONE 8, e75229 (2013).

This article was published online on 23 January 2019.
QUANTUM PHYSICS

\section{A traffic jam of light}

A technique that harnesses energy loss has been used to produce a phase of

matter in which particles of light are locked in place. This opens a path to realizing previously unseen exotic phases of matter. SEE ARTICLE P.51

\section{KADEN R. A. HAZZARD}

W hen light passes through matter, it slows down. Light can even be brought to a standstill when it travels through carefully designed matter. One way in which this occurs is when the velocity of individual particles of light (photons) in a material is zero. Another, more intriguing, way is when photons, which normally pass through each other unimpeded, are made to repel each other. If the repulsion is strong enough, the photons are unable to move, and the light is frozen in place. On page 51, Ma et al. ${ }^{1}$ report that such a phase of matter, known as a Mott insulator, can be produced by exploiting energy loss in a system in which photons move through an array of superconducting circuits.

A wide variety of experiments aim to engineer large quantum systems for use in computation and high-precision sensing, and to design materials that have unprecedented properties. These experiments often treat dissipation - the loss of energy or, more generally, information about a system - as anathema
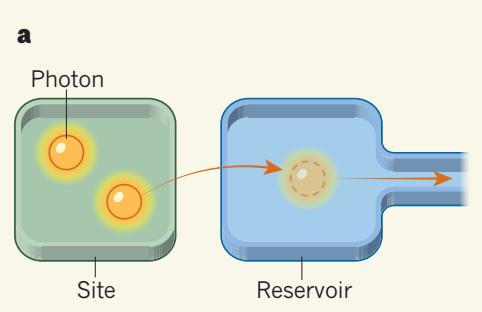

c

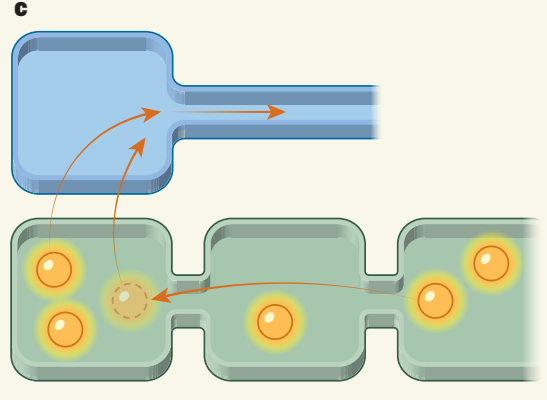

to producing large quantum systems. The reason is that tiny perturbations, including dissipation, often destroy quantum effects in systems of more than a few particles. Great care is therefore taken to minimize dissipation.

However, when dissipation is carefully engineered, it can also be a resource, and its utility for realizing exotic quantum matter is beginning to be harnessed ${ }^{2-8}$. One common way to use dissipation to produce unusual quantum states is to lower the temperature of a material. This can be accomplished by immersing the material in a coolant to extract energy. Just as cars on a motorway can go from a highenergy, smoothly flowing state to a jammed state by dissipating energy into heat and sound, quantum systems can go from a relatively hot initial state to a cold, jammed state by dissipating energy into photons.

Ma and colleagues used a more sophisticated method to dissipate energy from superconducting circuits, which are similar to ordinary circuits, but have some elements replaced by superconducting wires. The authors used a chain of eight superconducting

b

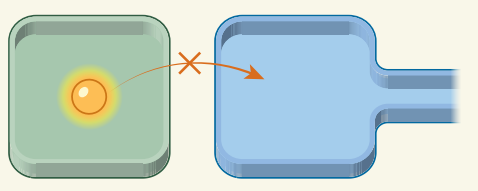

d

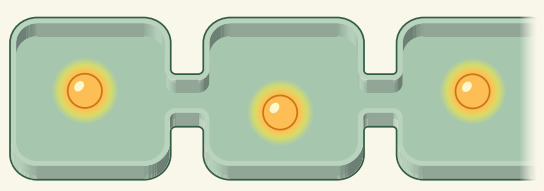

Figure 1 | Production of a Mott insulator. Ma et al. ${ }^{1}$ report a technique for organizing particles of light (photons) into a phase of matter called a Mott insulator. a, The method uses superconducting circuits that can be thought of as sites that photons can occupy. If there are two photons on a site, one photon can move between the site and a 'reservoir', and then will be rapidly lost to the outside world. $\mathbf{b}$, If there is a single photon on a site, it will not move to the reservoir. c, Coupling the end of a chain of sites to a reservoir forces the end site to be occupied by a single photon. If any of the other sites are occupied by two photons, one of the photons will move through the chain until it reaches the end site and then be lost to the reservoir. d, The end result of this process is a Mott insulator, a simple picture of which is a state that has exactly one photon per site. 
circuits called transmons. Each transmon can be thought of as a site that photons can occupy. If two photons occupy the same site, they interact with each other at a substantial energy cost.

The authors implemented two different schemes to produce a Mott insulator, a simplified picture of which is a state that has exactly one photon per site. Here, I will describe only the simpler scheme (although the second one was observed to yield a higher-quality final state). To understand this scheme, consider a single site, and how this site might be driven to be occupied by a single photon. It is easy to add a photon to an empty site, without dissipation, by applying a microwave-frequency electric field. However, such a process is equally likely to remove a photon from an already occupied site.

What is needed is an irreversible process that adds a photon to an empty site. Such a process can be engineered by combining nondissipative processes with a 'reservoir' that rapidly loses photons. In the authors' scheme, an applied microwave field causes photons to be added in pairs to a site (or subtracted in pairs, in the unavoidable reverse process). When the site is occupied by a pair of photons, it has the same energy as the reservoir and one photon can move to the reservoir and be lost, resulting in the site being left with a single photon (Fig. 1a). A single photon on the site is not energetically matched with the reservoir, and therefore remains on the site (Fig. 1b).

This idea extends to more than one site. In $\mathrm{Ma}$ and colleagues' scheme, the chain of sites in which the Mott insulator is to be prepared is attached at one end to a reservoir (Fig. 1c). If any site in the chain is doubly occupied, one of the photons from this site will wander through the chain until it reaches the end site. This photon will then move to the reservoir and be lost, ultimately resulting in a Mott-insulator state (Fig. 1d).

Although this simple picture makes it look as if the sites could be prepared independently to have exactly one photon, the actual Mottinsulator state is more complicated, and has quantum fluctuations that excite the system to include empty and doubly occupied sites. The authors' scheme prepares the system to include just the right excitations to be in the true Mottinsulator state. This state is an example of a strongly correlated phase of matter, which has been studied for decades in condensedmatter and ultracold-matter physics owing to its importance in quantum materials.

The strongly correlated state prepared in the current experiment is relatively simple, but the superconducting-circuit platform is flexible, and could be used to realize systems that have different geometries and different couplings between sites. Ma and colleagues' scheme is predicted to be able to prepare any gapped phase of matter - defined as having a non-zero energy cost to add or remove a particle - that occurs in any of these systems. One type of gapped phase that will surely be the target of future work is 'topological' matter. Fundamental open questions about the behaviour of topological matter can be explored in superconducting-circuit systems using dissipative preparation schemes ${ }^{9}$.

At least two further advances are needed if researchers are to use the authors' scheme to prepare complex quantum states. First, the technique needs to be extended to larger quantum systems. Second, the quality of state preparation needs to be increased. Currently, there is a relatively small (roughly 10\%) density of defects - sites that have either zero or two photons. Nevertheless, this density is at least ten times larger than that of comparable experiments that use, for example, ultracold atoms ${ }^{10}$. Reducing the defect density is challenging, but it seems to be an engineering issue rather than a fundamental one.

The ability to engineer quantum states promises to revolutionize areas ranging from materials science to information processing. Because quantum states are often as fragile as they are useful, robust state preparation will be essential to realizing their potential. Ma and colleagues' technique engineers dissipation to drive the system to the desired state and is therefore intrinsically robust to perturbations and noise. The robustness and generality of this scheme will ensure that, as it is refined, it will find a home in the quantum mechanic's toolbox.

Kaden R. A. Hazzard is in the Department of Physics and Astronomy and the Rice Center for Quantum Materials, Rice University, Houston, Texas 77005, USA.

e-mail:kaden.hazzard@gmail.com

1. Ma, R. et al. Nature 566, 51-57 (2019)

2. Kapit, E. Quantum Sci. Technol. 2, 033002 (2017)

3. Shankar, S. et al. Nature 504, 419-422 (2013).

4. Lin, Y. et al. Nature 504, 415-418 (2013)

5. Barreiro, J. T. et al. Nature 470, 486-491 (2011).

6. Fitzpatrick, M., Sundaresan, N. M., Li, A. C. Y., Koch, J. \& Houck, A. A. Phys. Rev. X 7, 011016 (2017).

7. Potočnik, A. et al. Nature Commun. 9, 904 (2018).

8. Hacohen-Gourgy, S., Ramasesh, V. V., De Grandi, C. Siddiqi, I. \& Girvin, S. M. Phys. Rev. Lett. 115, 240501 (2015).

9. Kapit, E., Hafezi, M. \& Simon, S. H. Phys. Rev. X 4, 031039 (2014)

10.Bakr, W. S. et al. Science 329, 547-550 (2010).

\section{An evolving picture of cell senescence}

DNA sequences called retrotransposons can copy themselves and reintegrate at new sites in the genome, causing damage. It now seems that inhibiting this process can prevent age-related health decline in mice. SEE ARTICLE P.73

\section{BENNETT CHILDS \& JAN VAN DEURSEN}

( $\mathrm{ld}^{1}$ and diseased ${ }^{2}$ tissues often contain cells that have entered a state called senescence, in which they stop dividing and become resistant to death-inducing pathways. These cells secrete a collection of factors, collectively known as the senescenceassociated secretory phenotype (SASP), that have inflammatory, protein-degrading and other biologically active properties, and can impair tissue function. There is therefore interest in targeting the SASP to combat agerelated diseases. The composition of the SASP varies, and might change over the lifetime of the senescent cell ${ }^{3}$. However, the molecular drivers involved in this evolution are incompletely understood. On page 73, De Cecco et al. ${ }^{4}$ identify a key contributor to the 'late' SASP: the reactivation of dormant DNA sequences called retrotransposons.

Retrotransposons are often called 'jumping genes', because the messenger RNA transcribed from them can undergo a process called reverse transcription to produce an identical DNA sequence that then reinserts into the genome at a different site. Although retrotransposons comprise about $42 \%$ of the human genome, most carry mutations that render them functionally inactive $e^{5}$. Transcription of those that remain functional must be prevented by protein- or RNA-based regulatory mechanisms to prevent the jumping of retrotransposons, which can cause either genetic mutations or genomic instability and might lead to cancer ${ }^{6}$. However, retrotransposons can be reactivated during ageing ${ }^{7}$.

De Cecco et al. found that one type of retrotransposon, LINE-1, was highly activated in senescent human cells within 16 weeks after they had stopped dividing - a stage the authors term late senescence. The group showed that high levels of the transcriptional repressor protein RB1 and low levels of the transcriptional activator protein FOXA1 normally keep LINE-1 in check. These proteins are abnormally expressed in late-senescent cells, enabling LINE-1 reactivation (Fig. 1).

At this late stage, the SASP is known to include two related inflammatory proteins called interferon- $\alpha$ and interferon- $\beta$. This signalling protein is part of an ancient antiviral mechanism called the cGAS-STING pathway, which is activated by the presence 